\title{
Analysis of Intravenous Urography in Renal Abnormalities in a Tertiary Healthcare Centre
}

\author{
Mohit Deswal ${ }^{1}$, Nitish Virmani2 ${ }^{2 *}$, Arshad Alam Khan², Ashita Jain ${ }^{2}$, Prashant Kumar Jha ${ }^{3}$, Mir Rizwan², B. B. \\ Sharma ${ }^{4}$, Aarzoo Tehlan ${ }^{1}$, Veenu Virmani ${ }^{5}$ \\ ${ }^{1}$ M.Sc Research Fellow, Radio-Imaging Technology, SGT University, Gurugram, Haryana, India \\ ${ }^{2}$ Assistant Professor, Radiation and Imaging Technology, Faculty of Allied Health Sciences, SGT University, Gurugram, \\ Haryana, India \\ ${ }^{3}$ Tutor, Radio-Imaging Technology, Faculty of Allied Health Sciences, SGT University, Gurugram, Haryana, India \\ ${ }^{4}$ HOD \& Professor, Radio-diagnosis and Imaging Department, SGT University, Gurugram, Haryana, India \\ ${ }^{1}$ M.Sc Research Fellow, Radio-Imaging Technology, SGT University, Gurugram, Haryana, India \\ ${ }^{5}$ Freelancer Statistician, Manager, Orane International Gurugram, Haryana, India
}

*Address for Correspondence: Mr. Nitish Virmani, Assistant Professor, Radio-Imaging Technology, Faculty of Allied Health Sciences, SGT University, Gurugram, Haryana, India

E-mail: nitish.fahs@sgtuniversity.org

Received: 29 Dec 2020/ Revised: 12 Feb 2021/ Accepted: 15 Apr 2021

\begin{abstract}
Background: Intravenous urography is a useful technique and uses for the evaluation of pathology of the urinary tract with the help of ionizing radiation and contrast media. It includes control/scout film of the abdomen and posts contrast films at serial intervals. The study was to evaluate the role of IVU under fluoroscopy in different diagnostic conditions.

Methods: A quantitative prospective study conducted on 70 patients, who underwent the IVU procedure. Consent form, History and symptoms were obtained from the patient before the investigation.

Results: A total of 70 patients were added to this study out of which 65 patients were found with positive findings and 5 were reported under normal study. Out of a total (65), 44 patients were found normal functioning right kidney and 21 have delayed functioning. And out of total (65) 46 normal functioning of left kidney, 14 delayed and 5 non-functioning left kidneys. Pathological finding through Intravenous urography was Right renal calculus in 25 patients, right hydronephrosis in 35 patients, left renal calculus in 15 patients and left hydronephrosis in 27 patients. Left PUJ calculus is shown in 7 patients, Left VUJ calculus is in 9 patients and left ureteric calculi are seen in 12 patients.

Conclusions: The study concludes uses of IVU should not be considered as a primary investigation for renal anomalies. Ultrasonography should be performed as a primary investigation. Patient with long-standing symptoms is more likely to diagnosed pathology in IVU. For the radiolucent calculus, IVU found gold standard procedures as compared to sonography.
\end{abstract}

Key-words: IVU, IVP, Inflammation, Infection, Urography, UTI, Urinary tract

\section{INTRODUCTION}

Intravenous Urography/ Intravenous Pyelography (IVU/ IVP) is a useful technique used for the evaluation of pathology of the urinary tract.

\section{How to cite this article}

Deswal M, Virmani N, Khan AA, Jain A, Jha PK, et al. Analysis of Intravenous Urographyin Renal Abnormalities in a Tertiary Healthcare Centre. SSR Inst. Int. J. Life Sci., 2021; 7(3): 2801-2806.

Access this article online https://iijls.com/
It uses ionizing radiation and contrast media for visualization of the urinary tract. It includes a control/scout film of the abdomen with kidney and urinary bladder and post-contrast films at serial intervals ${ }^{[1]}$.

Anatomy- The urinary system, also known as the renal system or urinary tract. This consists of kidneys, ureters, bladder, and urethra. The main purpose of the urinary tract is to remove waste from the body. Pair of kidneys are located between the dorsal body wall and parietal peritoneum on both sides (left and right sides). 
The formation of urine begins in the functional unit of the kidney that is called the nephrons. Urine then flows through a system of conversing tubules called collecting ducts. From there urine continues its flow into the ureter, transporting urine into the urinary bladder. Urine exits through the external urethral meatus ${ }^{[1]}$.

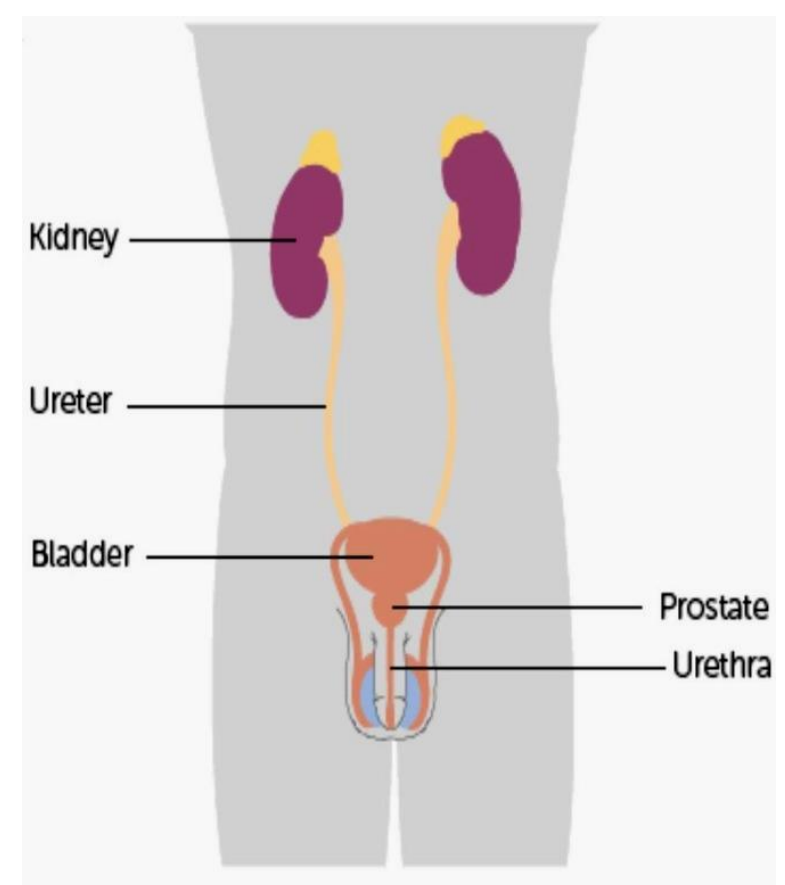

Fig. 1: Picture depicting anatomy of urinary tract

Intravenous Urography- The IVU procedure is performed under fluoroscopy to evaluate the urinary tract and for the procedure, the patient should void before the examination. If there is any calcification in the urinary tract, the location will assess before the injection of contrast medium, because contrast obscure calcification. For the position and nature of calcification, oblique conventional radiographs can be helpful ${ }^{[1,2]}$. Plain films are obtained first and radio-opaque calculi are identified. Urographyprograms are produced by filteration of contrast material by the nephrons, with the images of renal parenchyma 1-3 minutes after contrast medium injection. When the obstruction is the issue, a standard radiograph is collimated to the kidney, it may be sufficient in young patients. Oblique radiographs may be of added benefit if findings allow lateralization ${ }^{[2]}$. If we talk about the size of the kidney, it should be assessed on every radiograph, and best visualization during the nephrographic phase. An image is collimated to the kidneys to detect renal calculus and visualization of collecting systems which are obtained in 5 minutes after the compression device is applied. Oblique images are helpful for optimal evaluation of the ureters and pelvicalycealsystems. When compression is released, it allows the delivery of contrast material into the ureters [3].

KUB radiograph is obtained immediately after compression release, which is 15 minutes after administration of the contrast medium. Imaging with the patient in the prone assists with visualization of radiopaqueparts of the ureters, especially, when there is an obstruction. In such cases, delayed images must be an important tool and must take, until opacificationresults to the level of obstruction are not defined. 15-30 minutes after the administration of contrast media, the bladder is fullyfilled, and the 15minute KUB radiograph may be adequate for evaluation. Visualization of filling defects, oblique images may also be useful. Filling defects that are not related to enteric gas, which may project over the bladder on images obtained only in the anteroposterior projection ${ }^{[4-7]}$.

If there is specific attention is required to bladder disease, delayed images can be helpful and must be obtained to improve bladder distention, and oblique, prone, or post-void images may be obtained to evaluate filling defects. Delayed images of the bladder may be necessary if distention is incomplete ${ }^{[8]}$. The bladder position should be evaluated within the anatomy of the pelvis. Because the bladder is situated at the lower aspect of the anatomic pelvis, its position and appearance may be distorted by masses and other pathologic processes ${ }^{[9]}$. Bladder wall thickening and irregularity of the luminal contrast material associated with bladder defect. Early filling images followed by a post-void examination may help in the evaluation of filling defects.

The post maturation image is helpful in the diagnosis of patients with upper urinary tract dilatation and assessing residual volume when it is obtained immediately after voiding and demonstrates complete emptying of the bladder ${ }^{[10-12]}$.

Need of the study- IVU is usually not preferred over ultrasound and CT Urography due to many drawbacks. On USG, ureteric evaluation is often limited due to overlying bowel gases. CT Urography has its disadvantages like high radiation dose and high cost of the examination. Hence in a rural setting with the majority of patients belonging to low socioeconomic groups, IVU is still a preferred, relatively cheaper and 
valuable technique.

\section{MATERIALS AND METHODS}

This quantitative prospective study was carried out to assess the role of intravenous urography in renal abnormalities on the patients, who visit the radiology department of SGT Hospital and Research Institute, Gurugram, Haryana. This study was conducted over 6 months from $1^{\text {st }}$ October 2019 to $1^{\text {st }}$ April 2020. A total of 40 patients were selected pre-decided according to the inclusion criteria and exclusion criteria of this study. The consent form of each patient was acquired. History and symptoms were obtained from the patient before the investigation. IVU was performed under fluoroscopy using intravenous contrast media.

Exclusion criteria- Patients with a kidney transplant, Postoperative patients, patients with malignancies, patients with ongoing chemo/radiotherapy are excluded from the study to avoid putting any patient at risk of delayed urgent care.

Inclusion criteria- Patients with the following findings in ultrasonography were included in this study: Hydronephroticpatients, Renal Calculi, Congenital abnormalities, Horseshoe kidney, absent kidney.

Ethical approval- Ethical approval was taken from the central institutional committee of SGT University; Hospital \& Research Institute. Informed consent was obtained from all the participants.

Statistical analysis- Statistical analysis for this article like frequency and average was calculated through MS Office 2009.

\section{RESULTS}

A total of 70 patients were added to this study out of which 65 patients were found with positive findings and 5 were reported under normal study (Fig. 2). Out of a total (65), 44 patients were found normal functioning right kidney and 21 have delayed functioning (Fig. 3). And out of total (65) 46 normal functioning of left kidney, 14 delayed and 5 non-functioning left kidneys (Fig. 4). Pathological finding through Intravenous urography was Right renal calculus in 25 patients, right hydronephrosis in 35 patients, left renal calculus in 15 patients and left hydronephrosis in 27 patients (Fig. 5). Left PUJ calculus is shown in 7 patients, Left VUJ calculus is in 9 patients and left ureteric calculi are seen in 12 patients.

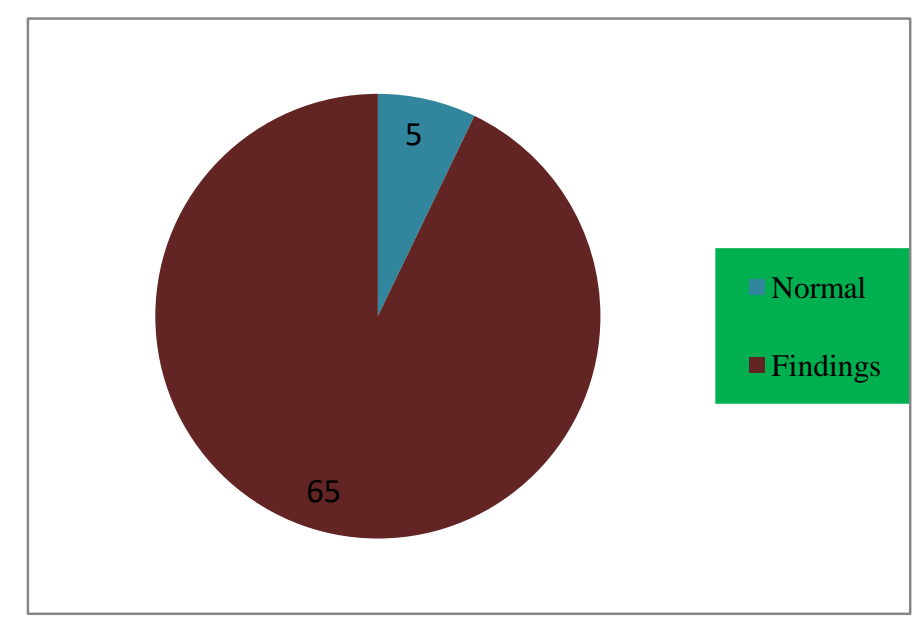

Fig. 2: Shows findings in IVU

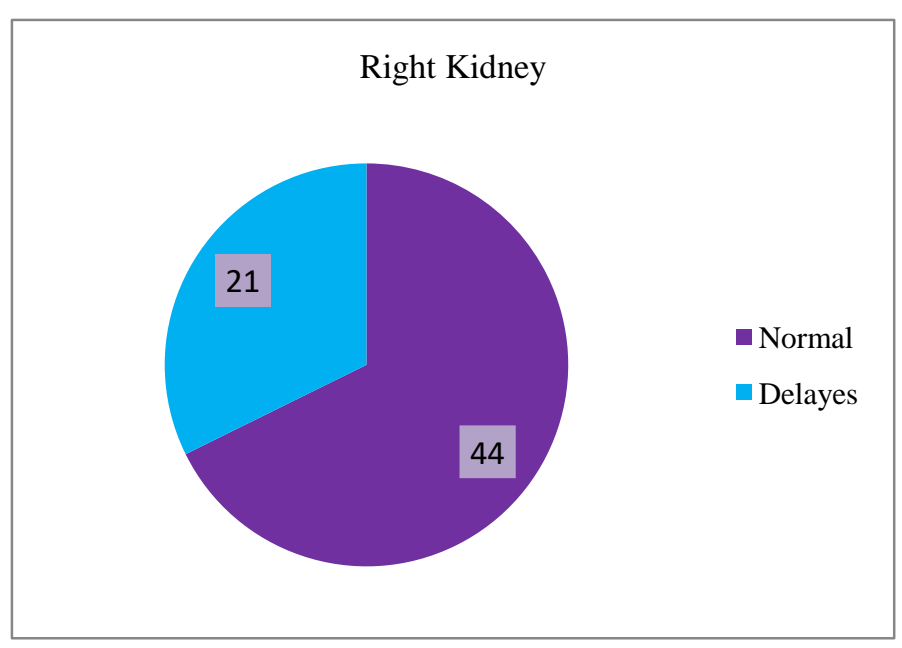

Fig. 3: Shows functioning of right kidney

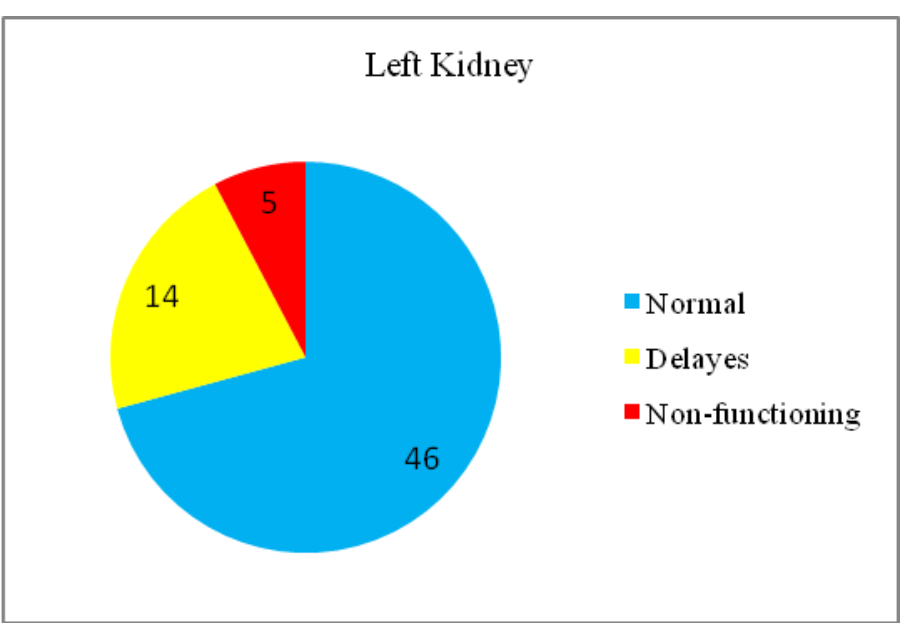

Fig. 4: Shows functioning of left kidney 


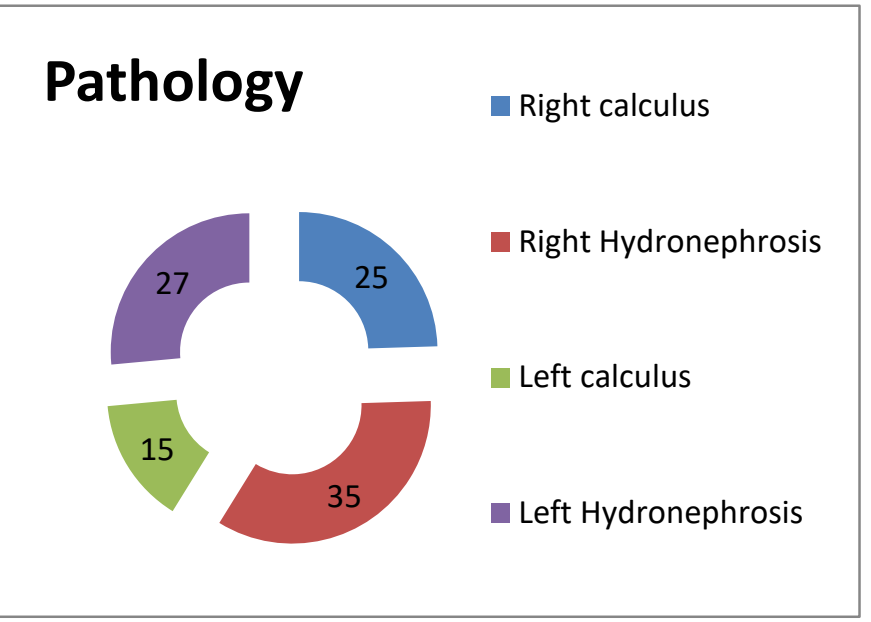

Fig. 5: Shows pathology in IVU

The result of this study shows intravenous urography is the gold standard evaluation of physiological functioning of the kidney means rather the kidney's excretion function is normal or delayed or not functioning. Clean means is intravenous urography can be used how well kidney, ureters and bladder are in good functioning. Anatomical and physiological details are well evaluated in the urography procedure. The evaluation of hydronephrosis is easy and rapid evaluation in intravenous urography. Calculus in PUJ (pelvic ureteric junction) and ureterovesical junction (VUJ) with contrast hold-up are well diagnosed in the intravenous urography that may replace the need for CT urography and can be used after the limitations of ultrasonography. Because PUJ and UVJ are cannot easily diagnose in ultrasonography where IVU are choice of imaging modality. In concern to ionizing radiation dose risk versus benefits, the ratio must be calculated. An IVU also will be helpful for diagnosis difference between urinary tract obstruction and calculus it may also help the physician to choose rather go for medication only or further invasive procedure.

\section{DISCUSSION}

This study suggested that a non-ionizing procedure Intravenous Urography is found the gold standard investigation to know the physiological functioning of kidneys for kidneys and it is the most common choice of procedure in tertiary health care centres, rather studies have proven role of sonography is more a primary diagnostic of renal diseases [9]. Also, it is important the selection of choice of the procedure according to the clinical or previous medical history of each patient. For each pathology imaging modality varies like in nephrolithiasis condition computed tomography is choice of selection versus ultrasonography ${ }^{[13]}$ and for different pathology patient consideration changes and management of patient must be done as per guidelines for the particular disease those may also vary from patient to patients ${ }^{[11]}$. In a comparison of computed tomography versus ultrasound versus intravenous urography, ultrasonography considers as a fast-imaging technique with a rapid result. Also, after sonography clinicians can make the easy call for the next choice imaging modality ${ }^{[12]}$. Sonography equipment has a mobile facility that is easy to use in emergencies department, Intensive care units, operation theatres; those are addon in the benefits of fast imaging and rapid result ${ }^{[14,15]}$. Studies do not suggest that Intravenous Urography should be the first choice of selection procedure but it play an important role right after the sonography limitations.

The study of upper urinary tract obstruction studies shows different appearances of ureters in different imaging modality could give a better diagnosis to patient bit simultaneously it also increases the time and cost effects for the patients. Hereby intra-venous urography has the advantage for the primary selection of procedure after the limitations of conventional X-Ray Kidney ureter and bladder ${ }^{[16-19]}$. Congenital diseases like horseshoe kidney, one kidney or congenital hydronephrosis are difficult to diagnose and treat in early phases also studies have proven urinary monocyte chemotactic protein-1 osteopontin and regulated upon activation, normal T-cell and secreted are useful in early diagnosis of congenital diseases. Whereas these markers are also shown dangerous effects to renal and lead to nephropathy too, hereby laparoscopy shows promising result by Taranta-

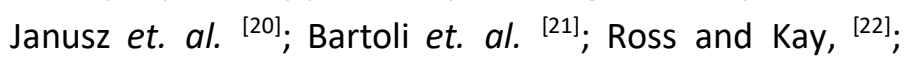
Yurkanin et. al. ${ }^{[23]}$. Hereby study suggests intravenous urography can lead to a minimally invasive procedure. That can also help to better management of the patient and to plan a line of treatment. So that physicians can show promising healthcare to patients ${ }^{[24,25]}$.

\section{CONCLUSIONS}

The study concludes uses of IVU should not perform as a primary investigation to all patients. Ultrasonography must be performed as a primary investigation. Duration of symptoms must be taken into the account. A patient with long-standing symptoms is more likely to diagnose with pathologies in IVU. For the radiolucent calculus, IVU 
found a gold standard procedure as compared to sonography. IVU also shows the functioning of the kidneys and any obstruction in the tract.

The study shows the uses or importance of IVU in diagnostic radiology as it visualises any pathologies or obstruction, which leads to more difficulties for the patient in future.

\section{ACKNOWLEDGMENTS}

We are thankful to Dr. Abhivind Bhutani (PG Resident), Ankit Kumar and Dhiraj M.Sc. fellows for data collection.

\section{CONTRIBUTION OF AUTHORS}

Research concept- Mr. Mohit Deswal, Mr. Nitish Virmani

Design \& Materials- Mr. Mohit Deswal, Mr. Nitish Virmani

\section{Supervision- Mr. Nitish Virmani}

Data collection- Mr. Prashant Jha

Data analysis and Interpretation- Ms Aarzoo, Ms. Veenu Literature search \& Critical review- Mr. Arshad Alam Khan

Writing article- Mr. Mohit Deswal

Article editing- Ms. Ashita Jain

Final approval- Dr. BB Sharma, Dr. Mir Rizwan

\section{REFERENCES}

[1] Dietrich CF. Efsumb, Course Book on Ultrasound. EFSUMB. London, UK, 2012.

[2] O'Neill WC, Robbin ML, Bae KT, et. al. Sonographic assessment of the severity and progression of autosomal dominant polycystic kidney disease: The consortium of renal imaging studies in polycystickidney disease (CRISP). Am J Kidney Dis., 2005; 46: 1058-64.

[3] Meister M, Choyke P, Anderson C, et. al. Radiological evaluation,management, and surveillance ofrenal masses in vonhippel-lindau disease. Clin Radiol., 2009; 64: 589-600.

[4] Leveridge MJ, Bostrom PJ, Koulouris G, et. al. Imagingrenal cell carcinoma withultrasonography, CT and MRI. Nat Rev Urol., 2010; 7: 311-25.

[5] Tamai H, Takiguchi Y, Oka M, et. al. Contrastenhanced ultrasonography in the diagnosis of solid renal tumors. J Ultrasound Med., 2005; 24: 1635-40.

[6] Lou L, Teng J, Lin X, et. al. Ultrasonographic features of renal oncocytoma with histopathologiccorrelation. J Clin Ultrasound, 2014; 42: 129-33.
[7] Yamashita Y, Ueno S, Makita O, et. al. Hyperechoicrenal tumors: Anechoic rim and intratumoral cysts in us differentiation of renal cell carcinoma from angiomyolipoma. Radiol., 1993; 188: 179-82.

[8] Clark TW, Millward SF, Gervais DA, et. al. Technology Assessment Committee of the Society of Interventional Radiology. Reporting standards for percutaneous thermalablation of renal cell carcinoma. J Vasc Interv Radiol., 2009; 20: 409-16.

[9] Mostbeck GH, Zontsich T, Turetschek K. Ultrasound of the kidney: Obstruction and medical diseases. Eur Radiol., 2001; 11: 1878-89.

[10]Chi AC, Flury SC, Urology patients in the nephrology practice. Adv Chronic Kidney Dis. 2013; 20: 441-48.

[11]Turk C, Petrik A, Sarica, K, et. al. Eau guidelines on diagnosis andconservative management of urolithiasis. Eur Urol., 2015.

[12] Nicolau C, Claudon M, Derchi LE, et. al. Imaging patients with renal colic-consider ultrasound first. Insights Imaging, 2015; 6: 441-47.

[13]Smith-Bindman R, Aubin C, Bailitz, J, et. al. Ultrasonography versus computed tomography for suspected nephrolithiasis. N Engl J Med., 2014; 371: 1100-10.

[14]Noble VE, Brown DF, Renal ultrasound. Emerg Med Clin N Am., 2004; 22: 641-59.

[15]Taha MA, Shokeir AA, Osman HG, et. al. Diagnosis of ureteropelvic junction obstruction in children: role of endothelin-1 in voided urine. Urol., 2007; 69(3): 56064.

[16]Shokeir AA. The diagnosis of upper urinary tract obstruction. BJU Int., 1999; 83(8): 893-900.

[17]Muthusami $P$, Ramesh A. Appearances of the circumcaval ureter on excretory urography and MR urography: A single-center case series. Indian J Radiol Imaging, 2013; 23(1): 81-85.

[18]Kaya C, Caliskan S. Comparison between intravenous urography and computed tomography urography in diagnosing ureteropelvic junction obstruction. Nephrourol Mon., 2012; 4(3): 585-86.

[19]Papachristou F, Pavlaki A, Printza N. Urinary and serum biomarkers in ureteropelvic junction obstruction: a systematic review. Biomarkers, 2014; 19(7): 531-40.

[20]Taranta-Janusz K, Wasilewska A, Debek W, et. al. Urinary cytokine profiles in unilateral congenital 
hydronephrosis. Pediatr Nephrol., 2012; 27(11): 2107-13.

[21]Bartoli F, Penza R, Aceto G, et. al. Urinary epidermal growth factor, monocyte chemotactic protein-1, and beta2-microglobulin in children with ureteropelvic junction obstruction. J Pediatr Surg., 2011; 46(3): 530-36.

[22]Ross JH, Kay R. Ureteropelvic junction obstruction in anomalous kidneys. Urol Clin North Am., 1998; 25(2): 219-25.
[23]Yurkanin JP, Fuchs GJ. Laparoscopic dismembered pyeloureteroplasty: a single institution's 3-year experience. J Endourol., 2004; 18(8): 765-69.

[24]Eden CG. Minimally invasive treatment of ureteropelvic junction obstruction: a critical analysis of results. Eur Urol. 2007; 52(4): 983-89.

[25]Dhillon HK. Prenatally diagnosed hydronephrosis: the Great Ormond Street experience. Br J Urol., 1998; 81 Suppl 2: 39-44.

\section{Open Access Policy:}

Authors/Contributors are responsible for originality, contents, correct references, and ethical issues. SSR-IIJLS publishes all articles under Creative Commons Attribution- Non-Commercial 4.0 International License (CC BY-NC). https://creativecommons.org/licenses/by-nc/4.0/legalcode (c) (i) (9) 